\title{
SIMULTANEOUS MINIMAX ESTIMATION OF PARAMETERS OF MULTINOMIAL DISTRIBUTION
}

Abstract. The problem of minimax estimation of parameters of multinomial distribution is considered for a loss function being the sum of the losses of the statisticians taking part in the estimation process.

1. Introduction. Let $X_{i}=\left(X_{i 1}, \ldots, X_{i r}\right), i=1, \ldots, m$, be observed by the $i$ th statistician. The random variables $X_{i}, i=1, \ldots, r$, have multinomial distribution with parameters $n_{i}, p=\left(p_{1}, \ldots, p_{r}\right)$, and are independent. The statisticians do not know the observations of their colleagues but they know all the numbers $n_{i}$. They cooperate with each other. The problem is to determine the simultaneous minimax estimator $d=\left(d_{1}, \ldots, d_{m}\right)$ of the parameter $p$ where $d_{i}\left(X_{i}\right)=\left(d_{i 1}\left(X_{i}\right), \ldots, d_{i r}\left(X_{i}\right)\right)$ is the estimator of this parameter, used by the $i$ th statistician. Let the loss function be

$$
L(p, d)=\sum_{i=1}^{m} k_{i} \sum_{j=1}^{r} c_{j}\left(d_{i j}\left(X_{i}\right)-p_{j}\right)^{2}
$$

where $k_{i}>0, c_{j} \geq 0$ are constants.

Let $R(p, d)$ be the risk function connected with the estimator $d$,

$$
R(p, d)=E_{p}(L(p, d))
$$

where $E_{p}(\cdot)$ is the operator of expected value with respect to the distribution of the random variable $X=\left(X_{1}, \ldots, X_{m}\right)$. Then we have to find the estimator $d^{0}$ for which

$$
\sup _{p} R\left(p, d^{0}\right)=\inf _{d} \sup _{p} R(p, d) .
$$

2000 Mathematics Subject Classification: Primary 62F15.

Key words and phrases: minimax estimation, multinomial distribution, Bayes estimator. 
2. Solution of the problem. Without loss of generality we can suppose that $c_{1} \geq \ldots \geq c_{r} \geq 0$. For the moment assume also that $c_{2} \neq 0$. Consider the estimator $d$ for which

$$
d_{i j}\left(X_{i}\right)=\frac{X_{i j}+\alpha_{j}}{n_{i}+\gamma}, \quad i=1, \ldots, m, j=1, \ldots, r .
$$

For this estimator the risk will take the form

$$
\begin{aligned}
R(p, d) & =\sum_{i=1}^{m} k_{i} \sum_{j=1}^{r} c_{j} E_{p}\left(\frac{X_{i j}+\alpha_{j}}{n_{i}+\gamma}-p_{j}\right)^{2} \\
& =\sum_{i=1}^{m} k_{i} \sum_{j=1}^{r} \frac{c_{j}}{\left(n_{i}+\gamma\right)^{2}}\left[E_{p}\left(X_{i j}-n_{i} p_{j}\right)^{2}+\left(\alpha_{j}-\gamma p_{j}\right)^{2}\right] \\
& =\sum_{j=1}^{r} c_{j} \sum_{i=1}^{m} \frac{k_{i}}{\left(n_{i}+\gamma\right)^{2}}\left[\left(\gamma^{2}-n_{i}\right) p_{j}^{2}+\left(n_{i}-2 \alpha_{j} \gamma\right) p_{j}+\alpha_{j}^{2}\right] .
\end{aligned}
$$

Assume that

$$
\sum_{i=1}^{m} \frac{k_{i}}{\left(n_{i}+\gamma\right)^{2}}\left(\gamma^{2}-n_{i}\right)=0 .
$$

It is easy to see that equation (4) always has a solution with respect to the constant $\gamma>0$.

Moreover assume that the constants $\alpha_{j} \geq 0$ satisfy the equations

$$
c_{j} \sum_{i=1}^{m} \frac{k_{i}}{\left(n_{i}+\gamma\right)^{2}}\left(n_{i}-2 \alpha_{j} \gamma\right)=c \quad \text { for } j \leq L,
$$

for some integer $L$ and

$$
\alpha_{j}=0 \quad \text { for } L<j \leq r .
$$

Finally, let

$$
\sum_{j=1}^{r} \alpha_{j}=\gamma
$$

From (4) and (5) we obtain for $j \leq L$, if $c_{j} \neq 0$,

$$
\sum_{i=1}^{m} \frac{k_{i}}{\left(n_{i}+\gamma\right)^{2}}\left(\gamma^{2}-2 \alpha_{j} \gamma\right)=\frac{c}{c_{j}}
$$

Then from (6)-(8), if $c_{L} \neq 0$, we obtain

$$
(L-2) \gamma^{2} \sum_{i=1}^{m} \frac{k_{i}}{\left(n_{i}+\gamma\right)^{2}}=\sum_{j=1}^{L} \frac{c}{c_{j}},
$$


or by (4),

$$
(L-2) \sum_{i=1}^{m} \frac{k_{i} n_{i}}{\left(n_{i}+\gamma\right)^{2}}=\sum_{j=1}^{L} \frac{c}{c_{j}} .
$$

Thus the constant $c$ is determined for given $L$ and $\gamma$.

Let $j_{0}$ be the greatest index $j$ for which $c_{j} \neq 0$ and let

$$
L=\max _{s}\left\{s \leq j_{0}: \sum_{l=1}^{s} \frac{1}{c_{l}}>\frac{s-2}{c_{s}}\right\} .
$$

We shall prove

LEMMA. For $j=L+1, \ldots, r$,

$$
q:=\frac{L-2}{\sum_{l=1}^{L} 1 / c_{l}} \geq c_{j} .
$$

Proof. Notice that the proof is only necessary for $j=L+1$. If $c_{L+1}=0$ the lemma obviously holds. If $c_{L+1} \neq 0$, from (10) it follows that

$$
L-1 \geq c_{L+1} \sum_{l=1}^{L+1} \frac{1}{c_{l}}=1+c_{L+1} \sum_{l=1}^{L} \frac{1}{c_{l}} .
$$

The lemma is a consequence of this inequality.

Taking into account (4) we obtain, from (3),

$$
\begin{aligned}
& R(p, d)=\sum_{j=1}^{r} c_{j} \sum_{i=1}^{m} \frac{k_{i}}{\left(n_{i}+\gamma\right)^{2}}\left[\left(n_{i}-2 \alpha_{j} \gamma\right) p_{j}+\alpha_{j}^{2}\right] \\
& \stackrel{(5),(6)}{=} \sum_{j=1}^{L} c p_{j}+\sum_{j=1}^{L} c_{j} \sum_{i=1}^{m} \frac{k_{i} \alpha_{j}^{2}}{\left(n_{i}+\gamma\right)^{2}}+\sum_{j=L+1}^{r} c_{j} \sum_{i=1}^{m} \frac{k_{i} n_{i}}{\left(n_{i}+\gamma\right)^{2}} p_{j} \\
& \stackrel{(9),(11)}{=} \sum_{i=1}^{m} \frac{k_{i} n_{i}}{\left(n_{i}+\gamma\right)^{2}}\left(\sum_{j=1}^{L} q p_{j}+\sum_{j=L+1}^{r} c_{j} p_{j}\right)+\sum_{j=1}^{L} c_{j} \sum_{i=1}^{m} \frac{k_{i} \alpha_{j}^{2}}{\left(n_{i}+\gamma\right)^{2}}
\end{aligned}
$$

Thus $R(p, d)=$ const $=C$ if $\sum_{j=1}^{L} p_{j}=1$ and always, by the Lemma, $R(p, d) \leq C$ for the simultaneous estimator $d$ defined by (2) and satisfying $(4)-(7),(9)$ and (10). On the other hand, for any $d$ and the loss function (1) the expected risk $r(\pi, d)=E(R(p, d))$ attains its minimum if

$$
d_{i j}\left(X_{i}\right)=E\left(p_{j} \mid X_{i}\right)
$$

Here $E(\cdot)$ is the expectation for a prior distribution $\pi$ of the parameter $p$ and $E\left(p_{j} \mid X_{i}\right)$ is the conditional expectation of $p_{j}$ given $X_{i}$. 
Let a prior distribution of $p=\left(p_{1}, \ldots, p_{r}\right)$ be defined as follows:

$$
\begin{aligned}
& P\left(p_{1}+\ldots+p_{L}=1\right)=1 \\
& g\left(p_{1}, \ldots, p_{L}\right)=\frac{\Gamma(\gamma)}{\Gamma\left(\alpha_{1}\right) \ldots \Gamma\left(\alpha_{L}\right)} p_{1}^{\alpha_{1}-1} \ldots p_{L}^{\alpha_{L-1}}
\end{aligned}
$$

where $g$ is a density. For the prior distribution (12) we obtain

$$
\begin{aligned}
& d_{i j}\left(x_{i 1}, \ldots, x_{i L}, 0, \ldots, 0\right)= E\left(p_{j} \mid X_{i 1}=\right. \\
&= \begin{array}{r}
x_{i 1}, \ldots, X_{i L}=x_{i L}, \\
\left.X_{i, L+1}=\ldots=X_{i r}=0\right)
\end{array} \\
&= \begin{cases}\frac{x_{i j}+\alpha_{j}}{n_{i}+\gamma} & \text { for } j=1, \ldots, L, \\
0 & \text { for } j=L+1, \ldots, r ; i=1, \ldots, m .\end{cases}
\end{aligned}
$$

Then the estimator $d$ defined by (2) and satisfying (4)-(7), (9), (10) is Bayes and from the Hodges and Lehmann theorem it follows that it is minimax.

For $r=2$ always $L=2, c=q=0$ and the relevant estimator is a constant risk estimator.

Up to this point we have assumed that $c_{2} \neq 0$. If only $c_{1} \neq 0$ the problem reduces to simultaneous estimation of the parameter $p$ of binomial distribution for the loss function

$$
L(p, d)=\sum_{i=1}^{m} k_{i}\left(d_{i}\left(X_{i}\right)-p\right)^{2}
$$

where $X_{i}$ is observed by the $i$ th statistician. In this case the simultaneous minimax estimator is given by the formula

$$
d_{i}\left(X_{i}\right)=\frac{X_{i}+\gamma / 2}{n_{i}+\gamma}
$$

where $\gamma$ satisfies (4).

The problem considered in this paper may be generalized by introducing the loss function

$$
L(p, d)=\sum_{i=1}^{m} k_{i} \sum_{j, l=1}^{r} c_{j l}\left(d_{i j}\left(X_{i}\right)-p_{j}\right)\left(d_{i l}\left(X_{i}\right)-p_{l}\right)
$$

where the matrix $\left\|c_{j l}\right\|_{1}^{r}$ is nonnegative definite. To solve the problem for this loss function one can apply linear programming methods as done by Wilczyński [2] for $m=1$.

For problems of minimax estimation of many parameters by one statistician see Trybuła [1]. 


\section{References}

[1] S. Trybuła, Some problems of simultaneous minimax estimation, Ann. Math. Statist. 29 (1958), 245-253.

[2] M. Wilczyński, Minimax estimation for multinomial and multivariate hypergeometric distribution, Sankhyā Ser. A 47 (1985), 128-132.

Institute of Mathematics

Technical University of Wrocław

50-370 Wrocław, Poland

Received on 6.2.2002;

revised version on 1.7.2002 